\title{
Leukemia Cutis in a patient with Chronic Myeloid Leukemia
}

\author{
Dr. K. Valarmathi ${ }^{1}$, Dr. A. Jamila ${ }^{2}$, Dr. Mary lily S. ${ }^{3}$, Dr. Subachithra T. ${ }^{4}$, \\ Dr. Deepa R. ${ }^{5}$ \\ ${ }^{1}$ (Department of pathology, Stanley medical college, Chennai, India) \\ ${ }^{2}$ (Department of pathology, Stanley medical college, Chennai, India) \\ ${ }^{3}$ (Department of pathology, Stanley medical college, Chennai, India) \\ ${ }^{4}$ (Department of pathology, Stanley medical college, Chennai, India) \\ ${ }_{5}^{5}$ (Department of pathology, Stanley medical college, Chennai, India)
}

\begin{abstract}
Leukemia cutis is defined as the infiltration of neoplastic leukocytes or their precursors into the epidermis, the dermis, or the subcutis which results in clinically identifiable cutaneous lesions. Here we describe a case of Chronic myeloid leukemia who presented with a subcutaneous skin nodule over the abdomen for which Fine needle aspiration cytology was done and was reported as leukemia cutis.
\end{abstract}

Keywords: Chronic myeloid leukemia, Fine needle aspiration cytology, Leukemia cutis

\section{Introduction}

Leukemia cutis (LC) is a term used for cutaneous manifestations of any type of leukemia. It has a wide range of cutaneous manifestations, which makes it difficult to clinically distinguish LC from other skin lesions. Patients with LC usually have systemic leukemia, but occasionally skin involvement can precede the involvement of the bone marrow or peripheral blood. Thus, a skin biopsy can be the first indication of the presence of leukemia in a subset of patients. The immunophenotyping of routinely processed skin biopsy specimens is useful in establishing the diagnosis of LC. Although the molecular pathogenesis of LC are not well defined, chemokine receptors and adhesion molecules may have an important role in skin tropism.

\section{II . Case History}

This case is about a $55 \mathrm{yr}$ old man who presented with anemia and abdominal mass . On examination he was found to have splenomegaly, hepatomegaly and a separate subcutaneous abdominal wall swelling(FIG 1). CT picture showed massive splenomegaly with a separate abdominal wall swelling( FIG 2).Peripheral blood examination was done and was reported as CML - chronic phase.RBC's were found to be normochromic with normoblasts. WBC's showed marked increase in count with increase in myeloid precursors . the blast percentage was found to be $5 \%$ and the basophil percentage was found to be $18 \%$ (FIG 3).

FNAC was done from the abdominal wall swelling.

Microscopic picture was cellular showing sheets of malignant cells composed of myeloblasts, promyelocytes, metamyelocytes, band forms and neutrophils in a hemorrhagic background.Thus a report of leukemia cutis was given(FIG 4,5).

\section{Discussion}

Leukemia cutis (LC) is a relatively rare condition and manifests in a variety of leukemia subtypes. Acute monocytic, myelomonocytic, and the T cell leukemias show the highest incidence (50 to 70\%) of LC. It is an uncommon manifestation of chronic myelocytic leukemia (2 to $8 \%$ ), and when it is present points towards blastic transformation of the disease. $(1,2)$ Although skin infiltrates have been reported in chronic phase of the disease, this either precedes or occurs simultaneously with a blood picture of more aggressive phase, hence the incidence largely corresponds to either the accelerated phase or the blast phase of the disease. $(2, \underline{3})$ It is noteworthy that as many as $90 \%$ of patients with LC may also have other extramedullary involvement, and as many as $40 \%$ may have meningeal or central nervous system involvement.(4)Leukemia cutis is more common in children than in adults and are most commonly associated with sub periosteal bone structures; the most common sites are skull, paranasal sinuses, sternum, ribs, vertebra and pelvis, lymph nodes and skin are also involved.(12)

The pathogenesis of LC is not so well defined. Chemokine receptors such as CCR4 and adhesion molecules such as cutaneous lymphocyte associated antigen (CLA) have been found to play a role in skin tropism of leukemic cells.(2)

Histologically, the tumor has three levels of differentiation : blastic, immature and differentiated.The blastic type is predominantly composed of myeloblasts with little evendence of maturation to the next stage.The myeloblasts have a moderate rim of basophilic cytoplasm, fine nuclear chromatin and two to four nucleoli. The 
immature type is the intermediate degree of differentiation containing principally myeloblasts and promyelocytes, eosinophil myelocytes are usually present.The differentiated type contains the promyelocytes and later stages of maturation. Eosinophil myelocytes are most abundant in this type.(12)

Leukemia cutis when present as the sole presenting feature of the disease without any peripheral blood manifestation, is described as aleukemic leukemia cutis.(5) In such cases in particular, cytochemistry with myeloperoxidase, naphthol ASD, chloroacetate esterase; immunohistochemical analysis using a panel of antibodies (LCA, CD 3, CD4, CD 5, CD8, CD13, CD19, CD33, CD43, CD45, CD56, CD68), in addition to cytogenetic [t (9:22), etc] and molecular analysis (RT-PCR for bcr-abl) of cells derived from the skin lesions can help establish the primary diagnosis. $(2,3, \underline{3})$

The skin lesions in LC show varied morphology and can be difficult to distinguish both clinically and histopathologically from nonspecific cutaneous lesions.(6) Lesions of LC are typically papules and nodules; indurated or hemorrhagic plaques, perifollicular acneiform papules, bullae, and palpable purpura. The lesions can range from red-brown to violaceous or plum color.(2,3) Other unusual manifestations include erythema nodosum, erythema annulare centrifugum, pyoderma gangrenosum and lesions mimicking urticaria, guttate psoriasis, vitiligo, stasis dermatitis and subungual leukemia cutis. $(\underline{3}, \underline{7}, \underline{8})$ It may also occur within established scars and within recent areas of trauma.(9) The varied clinical presentations of LC must be borne in mind so as to facilitate early diagnosis and timely treatment. Also, it is important to differentiate LC from sweet syndrome which is an acute febrile neutrophilic dermatosis found in patients of hematological malignancies and present as tender, erythematous, well demarcated papules and plaques and is successfully managed with steroids.(10)

In general, LC is a poor prognostic sign. Several studies indicate that the disease course is aggressive and the length of survival short when CML or AML is present with leukemia cutis.

A study by Kaddu et al. showed an average survival time of 9.4 months in CML with skin infiltrates.(3) Induction and consolidation chemotherapy followed by allogeneic bone marrow transplant can produce long survival and possibly cure. $(1,5)$

\section{References}

[1]. Ansell J, Bhawan J, Pechet L. Leukemia Cutis in Blastic Transformation of Chronic Myelocytic Leukemia: TdT Positive Blasts and Response to Vincristine and Prednisone. J Cut Path. 2006;7:302-9

[2]. Cho-Vega JH, Medeiros LJ, Prieto VG. Leukemia cutis. Am J Clin Pathol. 2008;129:130-42.

[3]. Kaddu S, Zenahlik P, Beham-Schmid C. Specific cutaneous infiltrates in patients with myelogenous leukemia: a clinicopathologic study of 26 patients with assessment of diagnostic criteria. J Am Acad Dermatol. 1999;40:966-78.

[4]. Baer MR, Barcos M, Farrell H. Acute myelogenous leukemia with leukemia cutis. Eighteen cases seen between 1969 and 1986. Cancer. 1989;63:2192-200.

[5]. Poventud MG, Fradera J, Pérez S. Aleukemic leukemia cutis preceding acute monocytic leukemia: a case report. P R Health Sci J. 2008;27:256-8.

[6]. Ratnam KV, Khor CJ, Su WP. Leukemia cutis. Dermatol Clin. 1994;12:419-31.

[7]. Newman MD, Milgraum S. Leukemia cutis masquerading as vitiligo. Cutis. 2008;81:163-5.

[8]. Papadavid E, Panayiotides I, Katoulis A. Stasis dermatitislike leukaemic infiltration in a patient with myelodysplastic syndrome. Clin Exp Dermatol. 2008;33:298-300.

[9]. Burns CA, Scott GA, Miller CC. Leukemia cutis at the site of trauma in a patient with Burkitt leukemia. Cutis. 2005;75:54-6.

[10]. Cohen PR, Kurzrock R. Chronic myelogenous leukemia and sweet syndrome. Am J Hemat.2006;32:134-7.

[11]. EXTENSIVE CUTANEOUS MANIFESTATIONS: PRESENTING FEATURE OF CHRONIC MYELOCYTIC LEUKEMIA IN SECOND BLAST CRISIS Manish Singhal, Sarika Singh, Rajive Kumar, and Vinod Raina, . Indian J Dermatol. 2010 JulSep; 55(3): 265-267.

[12]. 12.Rosai and Ackerman's surgical pathology, ninth edition.Juan Rosai..2004;2:2064-66.

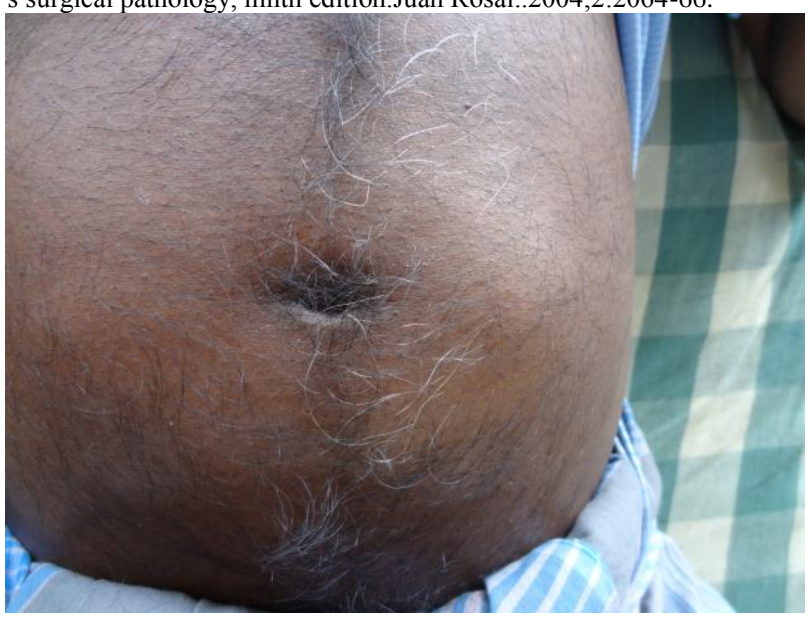

Figure 1: clinically the patient presented with abdominal wall swelling . on palpation was found to have splenomegaly, hepatomegaly and a separate abdominal wall swelling 


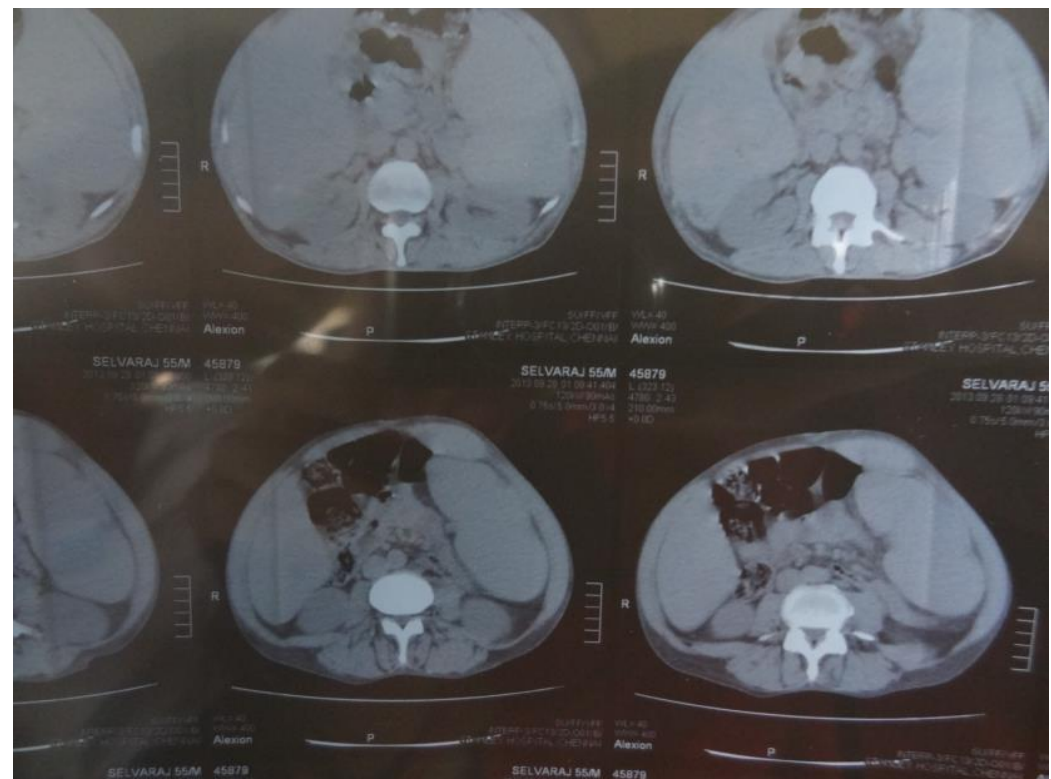

Figure 2 : CT picture ahowing splenomegaly with a separate abdominal wall swelling.

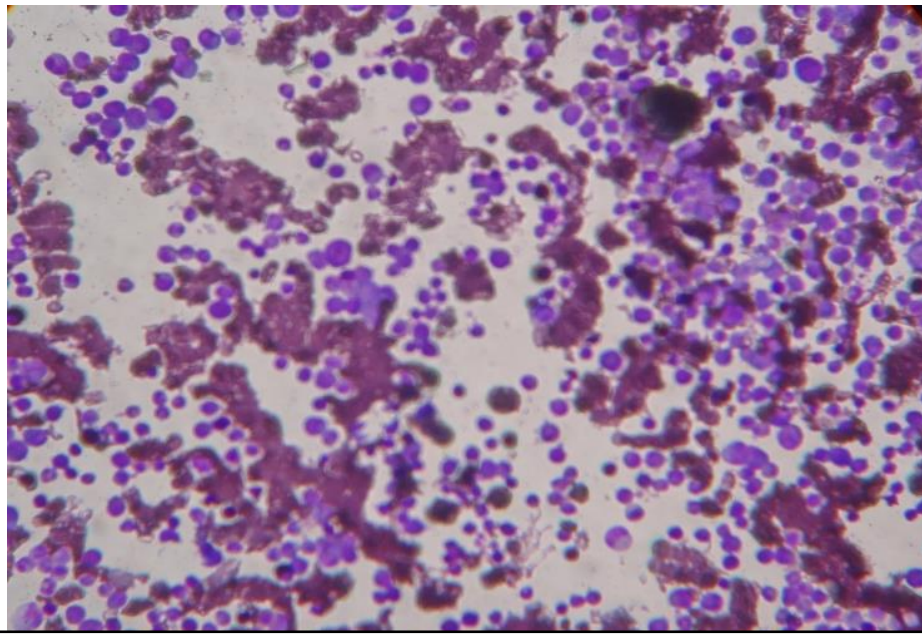

Figure 3: peripheral smear showing myeloid series in varying stages of maturation with a blast percentage of 5 $\%$. A diagnosis of CML - chronic phase was given.

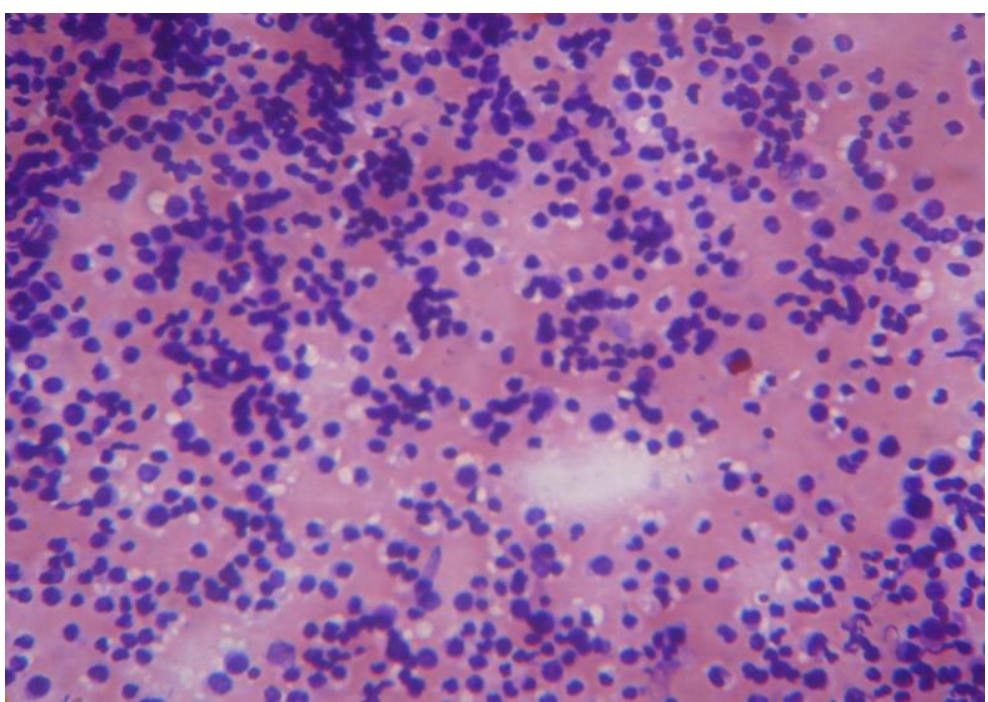




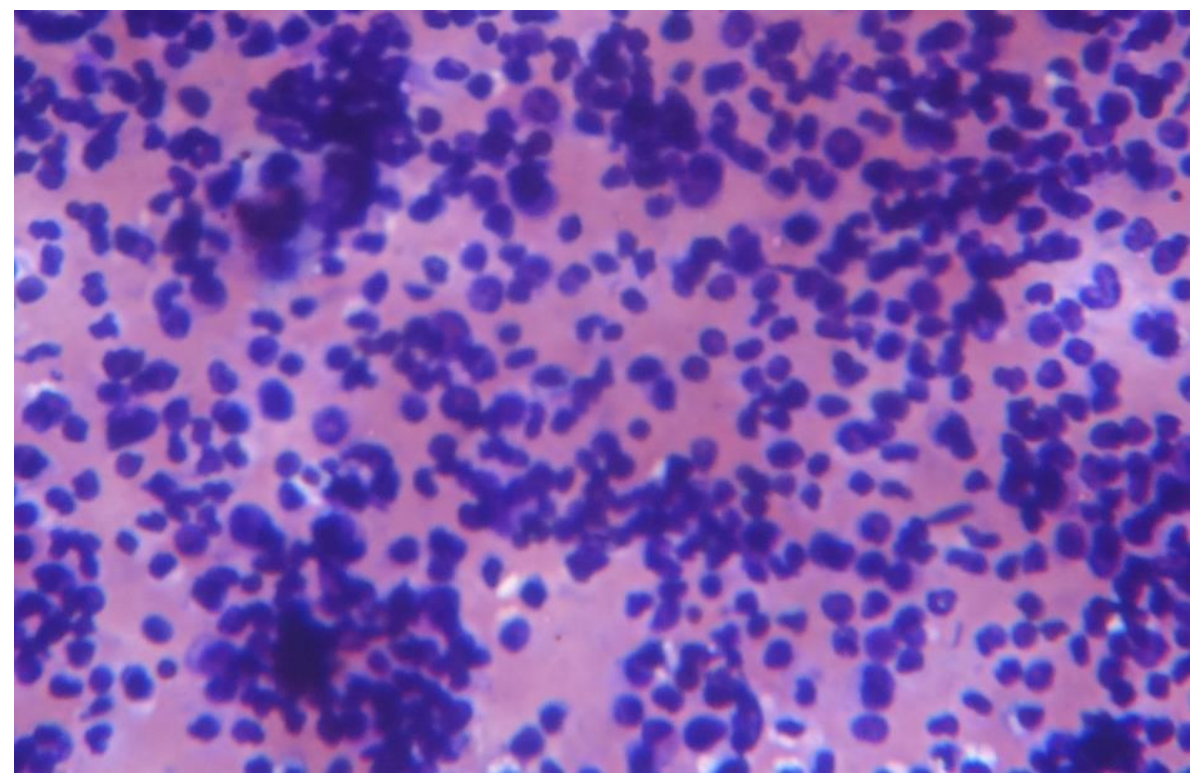

Figure 5 : high power picture showing leukemic cells in varying stages of maturation. Myeloblasts, promyelocytes, metamyelocytes, band forms and neutrophils seen. 